\title{
Recent refurbishment of the Oak Ridge Electron Linear Accelerator neutron source
}

K.H. Guber ${ }^{\text {a }, ~ T . S . ~ B i g e l o w, ~ C . ~ A u s m u s, ~ D . R . ~ B r a s h e a r, ~ J . A . ~ H a r v e y, ~ P . E . ~ K o e h l e r, ~ D . ~ W i a r d a, ~ R . B . ~ O v e r t o n, ~}$ J.A. White, and V.M. Cauley

Oak Ridge National Laboratory, P.O. Box 2008, Oak Ridge, TN 37831-6356, USA

\begin{abstract}
For almost 40 years the Oak Ridge Electron Linear Accelerator (ORELA) at Oak Ridge National Laboratory (ORNL) has been operational and has provided high-resolution neutron cross-section data for many isotopes in the energy range from thermal up to $60 \mathrm{MeV}$. ORELA is a four-section radiofrequency electron linear accelerator with a water-cooled tantalum neutron production target. Short (2-30 ns) electron burst widths together with available long flight paths lead to excellent time-of-flight resolution. The electron beam energy can range up to $180 \mathrm{MeV}$ and at $50 \mathrm{~kW}$ of beam power yields a neutron production rate $10^{14} \mathrm{n} / \mathrm{s}$. We report on recent refurbishment activities that included improvements to the accelerator vacuum and electron gun design and other upgrades. The current ORELA program is focused on high-resolution neutron cross-section measurements for the Nuclear Criticality Safety Program for nuclides for which deficiencies in existing data have been identified. Additionally neutron cross-section measurements for nuclear astrophysics are performed to support studies of heavy element synthesis in Asymptotic Giant Branch stars. Detection and data analysis capabilities have been developed for making highly accurate measurements of neutron capture, neutron total, and (n, $\alpha$ ) cross sections simultaneously on different beam lines.
\end{abstract}

\section{Introduction}

The Oak Ridge Electron Linear Accelerator (ORELA) facility was developed to provide intense short neutron pulses for high-accuracy neutron cross-section measurements applying the time-of-flight (TOF) method. The cross-section data are used for modeling of nuclear power reactors, astrophysics, shielding, and other applications [1]. ORELA began operation in 1969 and has been providing high-resolution cross-section data for many nuclides over the energy range from $0.002 \mathrm{eV}-$ $60 \mathrm{MeV}$. The current ORELA program is focused on crosssection measurements for the Nuclear Criticality Safety Program and nuclear astrophysics. Detection and data analysis capabilities have been developed for making highly accurate measurements of neutron capture, neutron total, $(\mathrm{n}, \alpha)$, and fission cross sections simultaneously on different beam lines. A refurbishment program has led to improvements in the accelerator vacuum, electron gun, klystrons, interlocks, and other systems.

\section{Facility}

ORELA is equipped with a pulsed, gridded, electron gun (pulse width $2-30 \mathrm{~ns}$, repetition rate $1-1000 \mathrm{~Hz}$ ), a foursection radio frequency (RF) LINAC, and a water-cooled tantalum target with Be housing to generate short neutron pulses via $(\gamma, \mathrm{n})$ reactions. The RF power for the accelerator sections are provided by four 24-MW, 1300-MHz klystrons that can accelerate electrons up to $180 \mathrm{MeV}$ and yield a neutron production rate of $10^{14} \mathrm{n} / \mathrm{s}$ at $50-\mathrm{kW}$ beam power.

ORELA has 10 flight paths ranging in length from 9 to $200 \mathrm{~m}$ with 18 underground flight stations. The combination of

\footnotetext{
${ }^{a}$ Presenting author, e-mail: guberkh@ornl.gov
}

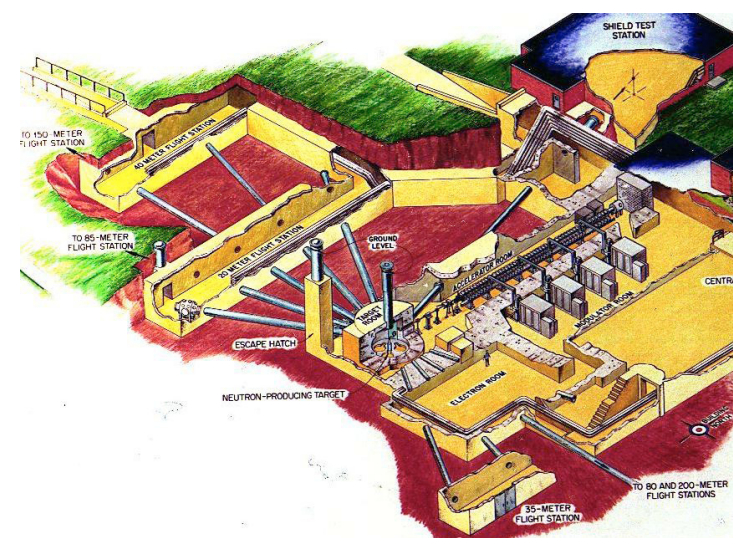

Fig. 1. Layout of the ORELA facility.

long flight paths and short neutron pulses leads to an excellent neutron energy determination using the TOF method. The water-cooled tantalum target is $15 \mathrm{~cm}$ in diameter and generates high-intensity pulses of neutrons with a comparatively small moderation time compared to other neutron facilities. Because of the relatively high flux and brightness of the source, a small sample size can be used for measuring neutroninduced cross sections. A layout of the ORELA facility is shown in figure 1 . Over the past 30 years, ORELA measurements have contributed to $\sim 80 \%$ of US Evaluated Nuclear Data File (ENDF/B) evaluations [1].

The ORELA accelerator [2], modulator, and control systems were designed and built by Varian in 1968 (Varian model V7727). The accelerator consists of four sections with an active length of $16.5 \mathrm{~m}$, which leads to an average accelerating gradient of $\sim 10 \mathrm{MeV} / \mathrm{m}$. Each section is a coupled cavity TM01 transmission line operating in the $2 / 3 \pi$ mode and is terminated with an internal RF load. Twelve water-cooling 
lines are distributed evenly around each section to maintain a constant operating temperature. A WR650 waveguide $\mathrm{SF}_{6}$ gas-cooled window is used to feed up to $24 \mathrm{MW}$ of microwave pulses into each section.

Each accelerator section is equipped with two 60-L/s Vacion pumps. In total 10 Vac-ion pumps are used to maintain a high vacuum. The electron gun has incorporated a specially designed 35-L/s Vac-ion pump.

\section{Refurbishment}

\subsection{Accelerator vacuum system}

Over the years, a number of vacuum issues have been a problem, including small leaks from the water cooling lines into the accelerator high vacuum. Unfortunately the leaks are in locations that are difficult to access and detect with a leak checker but could be roughly localized by observing relative responses on the various Vac-ion pump currents. Also, a residual gas analyzer (RGA) was installed on ORELA for better detection and identification of the leaks. For many years the leaks were controlled with a "stop leak" mixture circulated in the cooling water. The leak sealing material does eventually harden and block smaller water passages. In the last decade, this method of controlling leaks became less and less effective until the vacuum became too poor to effectively run the accelerator. By isolating the individual accelerator sections, two braze joints in the main water inlet of section 4 and cracks in threads of tapped water fittings on many of the outlets were found to be the major leak points.

During the original manufacturing process, the tapered water fitting threads were overcut, leading to small cracks between water-cooling and high vacuum systems. Eventually, the additives to the cooling water failed to work reliably, so a differentially pumped guard vacuum system was designed and installed on all outlet fittings of the cooling lines. This was achieved by installing O-ring sealed inserts that isolate the cracked threads from water. During the refurbishment period, this system was overhauled and improved by replacing the aging O-rings and installing new pumps with automatic valves to prevent oil-backstreaming. Because the leaks for two fittings, were rather large, individual pumps were used to maintain vacuum on those lines.

At the water inlets of accelerator section four, leaks were detected at a stainless steel to copper braze joint in the accelerator body. Several attempts to seal this leak with epoxy and other sealants were unsuccessful. Therefore, a mechanical seal with a compressed rubber gasket across the braze joint was designed and installed; it is still leak tight several month later.

All ten Vac-ion pumps were replaced, because they were at the end of their expected life time and had seen during this period a large gas load as a result of the vacuum problems. Also, the high-voltage cables and controls for the Vac-ion pumps were debugged and refurbished in the past year. In addition, a new 300-L/s turbo pump was purchased to facilitate startup of the Vac-ion pumps. The turbo pump also was very helpful to maintain a good vacuum for leak checking and operating the RGA.

\subsection{Klystrons}

Four high-power klystron tubes and associated modulators provide the microwave power pulses to drive the accelerator. The tubes used at ORELA are Litton model L-5081 1.3-GHz klystrons that produce up to 24-MW 2- $\mu$ s pulses with up to $-250 \mathrm{kV}, 250 \mathrm{~A}$ of beam power. A pulse repetition rate of up to $1000 \mathrm{~Hz}$ can be used; however, $525 \mathrm{~Hz}$ is typical for most experiments. Each klystron has a modulator unit that consists of an adjustable 0 - to $25-\mathrm{kV}$ high-voltage direct current $(\mathrm{HVdc})$ power supply and a thyratron-driven pulse-forming network (PFN). The thyratron/PFN system is setup with selectable pulse width and uses either a single or double thyratron depending on the pulse length. EEV CX7815 thyratrons are now used. A master oscillator, attenuator/phase shifter and traveling wave tube (TWT) amplifier system provides drive power for each klystron. A WR650 waveguide transmits power to each accelerator section. Flowing $\mathrm{SF}_{6}$ gas inside the waveguide is used to cool the klystron and accelerator vacuum windows and also to protect against breakdown at the windows.

The klystrons themselves have been quite reliable over the years and typically last several thousands of hours. A couple of tubes have been rebuilt and spares purchased. The modulator systems, especially the interlocks, cause the most down time. Water flow, air flow, under current monitors are intermittent at times. A high-speed trip system was used in the early days but disconnected due to excessive false trips. Also, voltage breakdown in the thyratron drive grid bias circuit during $\mathrm{HV}$ turn-off is currently an issue.

\subsection{Electron gun}

The ORELA electron gun shown in figure 2, is a triode structure in Pierce geometry [3]. It was developed and is built in house, because commercially available guns were not able to produce the required currents. The grid is a hexagonal web of molybdenum that is pulsed to about $1.5 \mathrm{kV}$ to generate a $\sim 24$ A pulse at $\sim 110 \mathrm{kV}$. Grid pulses are generated by an optically coupled thyratron-driven PFN pulser. The grid drive system and high-frequency cathode heater system are mounted in an $\mathrm{SF}_{6}$-filled gun tank. The pulse length can range from 2-30 ns, with $8 \mathrm{~ns}$ and 6 A being typical operating values.

The cathode material is a mixture of lacquer and $\mathrm{Ba}, \mathrm{Sr}$, and $\mathrm{Ca}$ carbonates that are converted into oxides. The material is electroplated on a Ni button [4]. This oxide type cathode

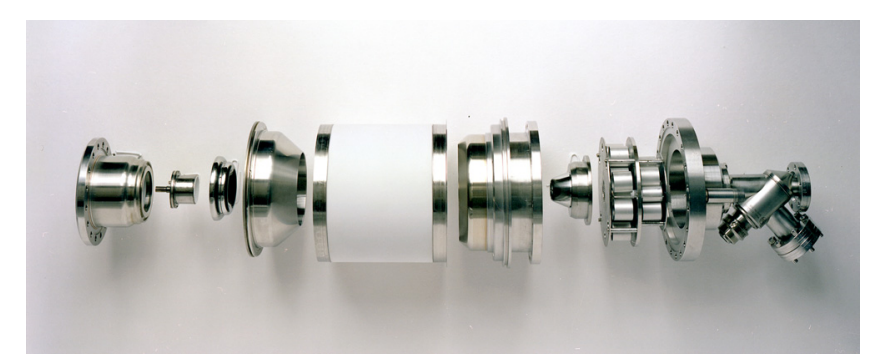

Fig. 2. Exploded view of the ORELA electron gun. The guns are built in-house. 


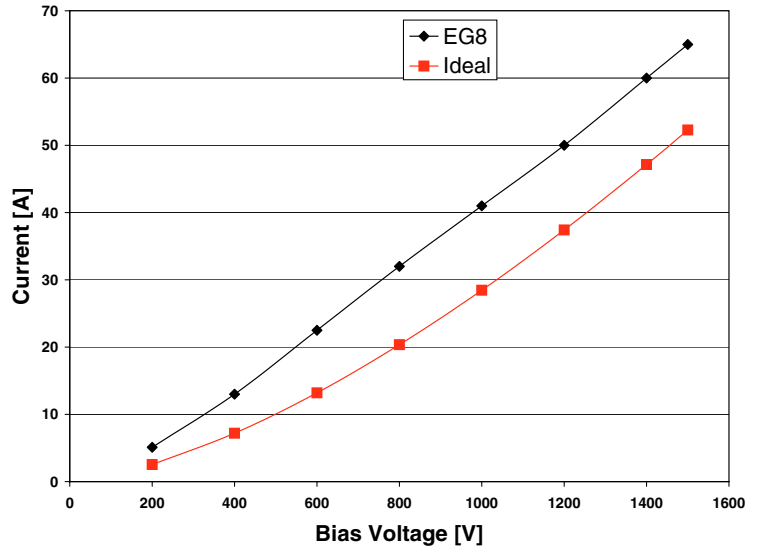

Fig. 3. Performance of electron gun EG8 cathode at 100-W heater power and a repetition rate of $100 \mathrm{~Hz}$.

was chosen because of its high emissivity at relatively low temperature $\left(\sim 850^{\circ} \mathrm{C}\right)$ to reduce the "dark" current thermally emitted by the grid.

Depending on the accelerator vacuum quality, the electron gun lasts typically more than $1000 \mathrm{~h}$. But a number of recent gun failures have been attributed to pin-hole vacuum leaks in the side of the insulator ceramic. It appears that this problem was due to scattered electrons from the anode region, so the design recently was changed to protect the ceramic. EGUN modeling of the gun potential distribution and emission was performed to investigate the effect of extending the cathode ring or the high-voltage bushings. One gun with an extended ring was built and performed acceptably. A second gun with an extended high-voltage bushing performed on the test stand above specification (see fig. 3) and showed very good highvoltage behavior. Also, the gun magnet alignment was improved to maximize coupling of electrons to the accelerator beam line and reduce anode overheating as a potential source of dark current emission.

Improved cathode processing and emitter materials have been investigated to increase gun current. The use of a new filament was investigated because the original failed quite often during operation at high temperature while converting the cathode. High-voltage processing up to $160 \mathrm{kV}$ is used to maintain stable operating conditions at the normally much lower operating voltage of about $110 \mathrm{kV}$. In addition, this procedure helps to minimize dark current.

\section{Conclusions and outlook}

OREL Auses a water-cooled tantalum target that generates a "white" neutron spectrum from thermal energies up to
$60 \mathrm{MeV}$. The water both cools the tantalum plates and moderates the generated neutrons. Up to $50 \mathrm{~kW}$ of beam power can be handled by the target; however $4-8 \mathrm{~kW}$ is typical for most experiments. Emanating out from the target room are ten evacuated neutron beam flight tubes. Several sets of collimators are used to define the neutron beams for each experiment. In addition, filters can be used at each flight tube to tailor the neutron energy spectrum and for background studies.

Since last year ORELA has new operators and engineers and has operated for more than 700 beam hours since refurbishment started. Further improvements in beam power are anticipated once more experience in tuning is gained and the system better understood. Reliability of vacuum repairs and aging modulator interlocks and controls are still factors; however, many improvements have been made to address these issues. Additional upgrades to the control system and upgrade of a data acquisition system are planned for the coming months.

In the past year, a number of cross sections have been measured at ORELA. Recent experiments include neutron capture and transmission measurements on a $99 \%$ enriched ${ }^{41} \mathrm{KCl}$ and a $\mathrm{Mn}$ sample, and ${ }^{64} \mathrm{Zn}(\mathrm{n}, \alpha)$. ORELA was operated at $525 \mathrm{~Hz}$ with a pulse width of $8 \mathrm{~ns}$ and a power 4 to $6 \mathrm{~kW}$ during these experiments.

A new capture apparatus is being developed on flight path 6 in the 40-m station. It is very similar to the apparatus on flight path 7 and will allow us to use the available running time more effectively. Also, a new transient-digitizer-based data acquisition system has been implemented for total crosssection measurements. This system improves data acquisition efficiency, allows more detailed data to be taken, and makes it possible to use different detector systems.

ORNL is managed by UT-Battelle, LLC for the US Department of Energy (DOE) under Contract No. DE-AC05-00OR22725. The work that is presented in this paper was sponsored by the DOE Nuclear Criticality Safety Program.

\section{References}

1. M.E. Dunn (ed.) Proceedings of the Oak Ridge Electron Linear Accelerator (ORELA) Workshop ORNL/TM-2005/272.

2. N.C. Pering, T.A. Lewis, Performance of a $140 \mathrm{MeV}$ High Current Short Pulse LINAC at ORNL, IEEE Trans. Nuc. Sci. NS-16, 316 (1969).

3. J.R. Pierce, Theory and Design of Electron Beams (Van Nostrand, 1954).

4. O.W. Christian, T.A. Lewis, ORNL/TM-7886, Oak Ridge National Laboratory, 1981. 